\title{
Is There a Racial Disparity in Coronavirus Disease 2019 Patients with Chronic Kidney Disease? An Experience in New York City
}

\author{
Takayuki Yamada ${ }^{1}$, Takahisa Mikami ${ }^{1}$, Nitin Chopra ${ }^{1}$, Hirotaka Miyashita ${ }^{1}$, Svetlana \\ Chernyavsky $^{1}$, and Satoshi Miyashita ${ }^{1}$ \\ ${ }^{1}$ Mount Sinai Beth Israel Hospital
}

April 28, 2020

Dear Editor,

Since December 2019, coronavirus 2019 (COVID-19) has spread worldwide. ${ }^{1}$ Some data have suggested that the prevalence and mortality of COVID-19 are different among races. ${ }^{2}$ However, this analysis did not account for potential confounding factors.

Since chronic kidney disease (CKD) is common, the number of COVID-19 patients with CKD will increase. However, there are scarce data about outcomes in CKD patients. We herein investigated the outcomes from COVID-19 in AAs compared to those in whites.

We analyzed Mount Sinai Health System (MSHS) medical records up to April 5, 2020, using Epic SlicerDicer software. We extracted data from patients who had positive for the COVID-19 reverse-transcription polymerase chain reaction (RT-PCR) test. Sex, age, race, and comorbidities (hypertension, diabetes mellitus, ischemic heart disease, heart failure, and atrial fibrillation) were extracted using the 10th revision of the International Statistical Classification of Diseases code. Mortality and intensive care unit (ICU) admission were tracked through April 12, 2020. Relative risks (RR) and $95 \%$ confidence interval (CI) in each race stratified by age groups and comorbidities were calculated using a Fisher's exact test. MSHS waived Institutional Review Board approval since this research used only deidentified, aggregate-level data.

During the study period, 1,269 AAs COVID-19 patients with 105 CKD patients and 1,450 whites COVID19 patients with 80 CKD patients were detected. AAs were younger (median 66, IQR 55-76) than whites (median 75, IQR 65-83) $(\mathrm{p}<0.001)$. There was no significant difference in mortality between AAs and whites (0.65 [0.36-1.15]). This tendency was observed after stratification by age and medical conditions. Similarly, AAs did not have an increased risk of ICU admission (0.84 [0.6-1.18])) even after stratification by age and comorbidities (Table).

To the best of our knowledge, this is the first study that compared the risk of severe outcomes among races in CKD patients. Although it has been suggested that there might be racial disparity in COVID-19, our study did not show any significant differences in outcomes, even after stratifying patients by age and comorbidities. Our data suggested that we do not need to stratify these patients by race.

The racial and ethnic diversity in NYC enabled us to investigate differences in outcomes among races in the same cohort. However, our study has several limitations. First, the number of patients was relatively small. Second, we did not access individual data, which prevented us from performing multivariate analyses. The fact that AAs were younger might mask differences among races.

In conclusion, AAs with CKD did not have a higher risk of mortality or ICU admission than whites with CKD. This trend was consistent after stratification by age, sex, or comorbidities. 
Acknowledgements: none

Conflict of Interest Disclosures: TY reports no conflict of interest. TM reports no conflict of interest. NC reports no conflict of interest. HM reports no conflict of interest. SC repots no conflict of interest. SM reports no conflict of interest.

Reference

1. Team CC-R. Preliminary Estimates of the Prevalence of Selected Underlying Health Conditions Among Patients with Coronavirus Disease 2019 - United States, February 12-March 28, 2020. MMWR Morb Mortal Wkly Rep. 2020;69(13):382-386.

2. Health N. Age adjusted rate of fatal lab confirmed COVID-19 cases per 100,000 by race/ethnicity group as of April 6, 2020 (Accessed Aprio 12, 2020). 2020.

\section{Hosted file}

Table.docx available at https://authorea.com/users/313875/articles/444273-is-there-a-racialdisparity-in-coronavirus-disease-2019-patients-with-chronic-kidney-disease-an-experiencein-new-york-city 